\title{
COMPARATIVO DE DOS MODELOS DE ESTILOS DE APRENDIZAJE EN UN GRUPO DE ESTUDIANTES DE QFB DE LA UACAM
}

\section{COMPARATION OF TWO LEARNING STYLE MODELS IN A GROUP OF QFB STUDENTS OF THE UACAM}

Marvel del Carmen Valencia Gutiérrez ${ }^{11}$

Magnolia del Rosario López MÉNDEZ ${ }^{21}$

María de Jesús García Ramírez ${ }^{31}$

Brillante Zavala Centeno ${ }^{41}$

Universidad Autónoma de Campeche ${ }^{1}$

San Francisco de Campeche, México

\section{RESUMEN}

En esta investigación se realizó un estudio de los estilos de aprendizaje a los estudiantes

1 mcvalenc@uacam.mx 9817504644 ORCID: 0000- 0002-3671- 0296

$2 \quad$ marlopez@uacam.mx 9811108014. ORCID: 0000- 0002- 7919- 894X

3 mjgarcia@uacam.mx 9821091426. ORCID: 0000 -0002- 2707- 8081

$4 \quad$ brzavala@uacam.mx 9811378916. ORCID: 0000-0001-7169-1724 que ingresaron en la generación 2018 del Programa Educativo de Químico Farmacéutico Biólogo de la Facultad de Ciencias Químico Biológicas de la Universidad Autónoma de Campeche. Se uso el modelo de Honey y Alonso también llamado CHAEA y el Modelo de la Programación Neurolingüística de Bandler y Grinder del canal Visual, Auditivo, Kinestésico, también llamado VAK. Los cuestionarios de las 
dos clasificaciones de estilos que se les aplicó a los 27 estudiantes que ingresaron en 2018, después de impartirles un curso de estilos, se les aplicaron nuevamente los instrumentos en 2019. Los resultados muestran que antes del curso 24 estudiantes aparentemente tuvieron un estilo preferencial específico considerando la clasificación de Honey y Alonso, después del curso solo 13 estudiantes se mantuvieron en un solo canal preferencial. Respecto al canal Visual, Auditivo, Kinestésico, también llamado VAK antes del curso 16 estudiantes tuvieron un canal preferencial, después del curso 18 estudiantes tuvieron un canal preferencial. De estos resultados fueron informados los estudiantes del Programa Educativo para que lo consideren en el desarrollo de sus estrategias de aprendizaje fortaleciendo su aprendizaje autónomo lo que contribuirá a incrementar el aprovechamiento escolar en el Nivel Superior.

PALABRAS CLAVE: Estilos, Aprendizaje, CHAEA, VAK

\section{ABSTRACT}

In this research work a study about learning styles was carried out, it was on the students whose freshman year was on 2018 of the educational program of Pharmaceutical Chemical Biologist in the School of Chemical Biological Sciences School of the Autonomous University of Campeche. The study considers the classification of Honey and Alonso, also called CHAEA and the Neurolinguistic Programming Model of Bandler and Grinder of the Visual, Auditive, Kinesthetic channel (VAK). The questionnaires of the two style classifications were applied to the 27 students who entered in 2018; then, after giving them a course about the styles, these instruments were reapplied to them in 2019. The results show that before the course, 24 students apparently had a specific preferential style considering the classification of Honey and Alonso; after the course only 13 students remained in a single preferential channel. Regarding the VAK, before the course 16 students had a preferential channel, after the course 18 students had a preferential channel. The students were informed of the results of the Educational Program, so they could consider them in the development of their learning strategies and therefore strengthening their autonomous learning, which will contribute to increasing the educational achievement in the undergraduate level.

KEY WORDS: Styles, Learning, CHAEA, VAK

\section{INTRODUCCIÓN}

Dentro de los grandes desafíos que hoy tienen la educación del siglo XXI y, en especial, la educación superior, se encuentran el reconocer y el potenciar al estudiante como el centro y el motor de su propio proceso formativo, lo cual requiere sumar esfuerzos no sólo por parte de las instituciones encargadas de la formación del educando que llega a sus aulas, sino también por parte del profesorado, que debe ser flexible y adaptable a las nuevas condicionantes que su actuación exige para la transferencia del conocimiento en las aulas ( Domínguez, 2015 ).

Los seres humanos tienen diferentes formas de percepción de la información, es desde estas que se alimenta el conocimiento a través de diferentes canales de percepción. El modelo VAK permite identificar el mejor de los tres canales de percepción: visual, auditivo, kinestésico. Independientemente de los canales perceptivos utilizados, diferentes estudios arrojan tendencias hacia algún canal por encima otro, la cantidad de información que el cerebro logra retener depende directamente de la metodología didáctica que el docente emplee, pues dependiendo de eso logrará estimular en mayor capacidad alguno de los tres canales perceptivos que se mencionan anteriormente (Reyes, 2017). 
Es importante mencionar que no se puede dejar de hacer alusión a la relación existente entre los Estilos de aprendizaje y el rendimiento académico. Un buen rendimiento académico es uno de los objetivos finales de todos aquellos que nos encontramos inmersos de alguna manera en los procesos de enseñanza y aprendizaje. Ello justifica todo lo que se ha estado explicando acerca del conocimiento sobre los Estilos de Aprendizaje de los estudiantes, así como la importancia de adaptación de la metodología docente a dichos estilos (Gutiérrez, 2018).

Los resultados encontrados por Castillo, plantean un reto importante para los docentes universitarios, ya que al ser los facilitadores del proceso enseñanza y aprendizaje es fundamental que tomen en cuenta las preferencias y características individuales de sus estudiantes, ya que, al tener conocimiento de tales diferencias en el aula de clase, pueden desarrollar así un amplio abanico de estrategias pedagógicas que propicien la adquisición de conocimientos significativos en los estudiantes (Castillo, 2015).

Por lo que es este estudio se realiza un diagnóstico de los estilos de aprendizaje considerando la clasificación de Honey y Alonso también llamado CHAEA y de los canales de percepción Visual, Auditivo, Kinestésico, también Ilamado VAK.

\section{ESTILOS DE APRENDIZAJE}

El término 'estilo de aprendizaje' se refiere al hecho de que cuando queremos aprender algo cada uno de nosotros utiliza su propio método o conjunto de estrategias. Aunque las estrategias concretas que utilizamos varían según lo que queramos aprender, cada uno de nosotros tiende a desarrollar unas preferencias globales. Esas preferencias o tendencias a utilizar más unas determinadas maneras de aprender que otras constituyen nuestro estilo de aprendizaje (ANUIES,2006).
La aplicación de los estilos proporciona un perfil de los estudiantes que sirve para actuar en dos direcciones (Escanero, 2013 citado en Escanero, 2016). Por una parte, para intentar mejorar los estilos de menor puntuación con el objetivo de que los alumnos posean elevadas puntuaciones en todos ellos, permitiéndoles que sean aprendedores eficientes en todos los contextos; por otra, para facilitar el conocimiento que permita la mejor y más rentable interacción metodológica para el aprendizaje. En ambos casos es preciso determinar, en primer lugar, el estilo de aprendizaje de los estudiantes (Escanero,2016).

Para el desarrollo de la investigación de González (2018), se centraron en el análisis de los cuatro tipos de estilos de aprendizaje de acuerdo a la forma de organizar y trabajar: estilo activo, reflexivo, teórico y pragmático (Aragón \& Jiménez, 2009 citado en González,2018). Sin embargo, es importante aclarar tal como lo expresaba Alonso (1992) citado en González 2018), no se dan estilos puros en las personas, sino perfiles de estilos de aprendizaje, así, los estudiantes con los predominios activo, reflexivo, teórico y pragmático poseen varias de las características principales que a continuación se describen:

Activo. Su aprendizaje es basado en la experiencia directa, por lo que se está siempre abierto a la experimentación, al trabajo en grupo, a los retos y se caracteriza por su implicación en la acción; quienes tienen preferencia por este estilo, se destacan por ser animadores, descubridores, improvisadores, creativos, líderes.

El estilo activo es característico de las personas que se implican plenamente y sin prejuicios en nuevas situaciones. Son de mente abierta, nada escépticos y se crecen ante los desafíos que suponen nuevas experiencias aburriéndose con las actividades a largo plazo. Son espontáneos, creativos innovadores, deseosos de aprender 
y resolver problemas (Aguilar,2010 citado en González, 2018).

La pregunta a la que responden en el aprendizaje los estudiantes con un estilo predominante activo es ‘¿Cómo?' De ahí que las situaciones favorables para ellos son cuando hay actividades que les presente un desafío. Realizar actividades cortas o de resultado inmediato. Cuando hay emoción, drama, crisis. Desfavorables, tener un papel pasivo, analizar o interpretar trabajos solos (Aguilar,2010 citado en González, 2018).

Reflexivo. Estilo basado en la observación desde diversas perspectivas, considera todas las alternativas; son ponderados, concienzudos, analíticos, detallistas, previsores, cuestionadores, investigadores.

El estilo reflexivo es característico de las personas que les gusta considerar las experiencias observadas desde diferentes perspectivas. Reúnen datos, analizándolos con detenimiento antes de llegar a una conclusión. Son prudentes y consideran todas las alternativas posibles antes de realizar un movimiento. Escuchan a los demás y no actúan hasta apropiarse de la situación.

La pregunta a la que responden en el aprendizaje los estudiantes con un estilo predominante reflexivo es ‘ ¿por qué?' De ahí que las situaciones favorables para ellos son cuando pueden ofrecer observaciones y analizar la situación. Cuando pueden pensar antes de actuar. Desfavorables, cuando se les fuerza a ser el centro de atención. Cuando se les apresura de una actividad a otra (Aguilar,2010 citado en González, 2018).

Pragmático: basado en la experimentación activa y búsqueda de Aplicaciones prácticas; se caracteriza por el eclecticismo; quienes desarrollan este estilo son experimentadores, prácticos, directos, realistas, eficaces; disfrutan llevando a cabo lo aprendido, planificando acciones, organizando, situándose en el presente, solucionando problemas (Aguilar,2010 citado en González, 2018).

En el estilo pragmático se busca la aplicación práctica de ideas. Son personas que les gusta actuar rápidamente y con seguridad con aquellas ideas y proyectos que les atraen, aprovechando la Primera oportunidad para experimentar. La pregunta a la que responden en el aprendizaje los estudiantes con un estilo predominante pragmático es "¿Qué pasaría sí? "De ahí que las situaciones favorables para ellos son cuando puede relacionar teoría y práctica, ver a los demás hacer algo. Desfavorables, cuando lo aprendido no se relaciona con sus necesidades inmediatas, con aquellas actividades que ni tiene una finalidad aparente, cuando lo que hacen no está relacionado con la realidad.

Por lo anterior, se hace evidente que una buena estructuración de los estilos de aprendizaje en los estudiantes universitarios puede facilitarle el acceso a un elevado volumen de información, adquirir conocimientos y orientarse en ellos con un verdadero sentido personal y capacidad creativa para la resolución de Los problemas que les plantea la sociedad (Aguilar,2010 citado en González, 2018).

Se definen cada uno de los canales de percepción como se muestra a continuación:

Visual: los sujetos que perciben desde este canal piensan en imágenes y tienen la capacidad de captar mucha información con velocidad, también son capaces abstraer y planificar mejor que los siguientes estilos. Aprenden con la lectura y presentaciones con imágenes.

Auditivo: los sujetos que utilizan el canal auditivo en forma secuencial y ordenada aprenden mejor cuando reciben explicaciones orales y cuando pueden hablar y explicar determinada información a otra persona. Estos alumnos no pueden olvidar una palabra porque no saben cómo sigue la oración; además, no permite 
relacionar conceptos abstractos con la misma facilidad que el visual. Es canal es fundamental en estudios de música e idiomas.

Kinestésico: son sujetos que aprenden a través de sensaciones y ejecutando el movimiento del cuerpo. Es el sistema más lento en comparación a los anteriores, pero su ventaja es que es más profundo, una vez que el cuerpo aprende determinada información le es muy difícil olvidarla; así, estos estudiantes necesitan más tiempo que los demás, lo que no significa un déficit de comprensión, sino solo que su forma de aprender es diferente (Reyes, 2017).

Observar el comportamiento de nuestros alumnos nos puede dar mucha información sobre su manera preferida de aprender. Nuestra manera de pensar y de procesar la información se refleja en nuestro comportamiento. En el cuadro los Sistemas de Representación y el comportamiento se dan algunas indicaciones generales sobre el tipo de comportamiento normalmente asociado a los distintos sistemas de representación (Neria, 2015).

\section{METOdOLOGÍA}

A continuación, se describe en forma la metodología seguida para este estudio:

Población de estudio: esta población fue de 27 estudiantes pertenecientes al año de ingreso 2018, del Programa Educativo de Químico Farmacéutico Biólogo de la Facultad de Ciencias Químico Biológicas de la Universidad Autónoma de Campeche

Sujetos: en esta investigación se tuvo un total de 27 estudiantes, de los cuales son 12 mujeres y 15 hombres con un rango de edad de 18 a 21 años.

Los instrumentos utilizados en el trabajo fueron los siguientes:
1.- Encuesta de estilos de aprendizaje del autor

P. Honey y A. Mumford (CHAEA) (Alonso, 1994).

2.- Test de Sistema de Representación Favorito del Modelo de la Programación Neurolingüística de Bandler y Grinder. Este test Visual, Auditivo, Kinestésico (VAK) es para ayudar a descubrir la manera preferida de aprender. Cada persona tiene su manera preferida de aprender. Reconocer las preferencias ayudará a comprender las fuerzas en cualquier situación de aprendizaje (Robles, 2000).

Procedimiento: En agosto de 2018 se aplicó el pretest a los 27 estudiantes que ingresaron en 2018, posteriormente se les impartió un curso de 4 horas, sobre los modelos de aprendizaje en dos sesiones, utilizando como material didáctico diapositivas, señalando en este material la teoría respectiva de los dos modelos, como el modelo de aprendizaje de Honey -Alonso, el Modelo de la Programación Neurolingüística de Bandler y Grinder, durante el desarrollo del curso de la unidad de aprendizaje que se imparte en el semestre de la fase 1 de ciclo escolar, se les va señalando en cada actividad la relación de estas con los estilos de aprendizaje que utilizan los estudiantes de forma cotidiana en el aula, se les da ejemplos también en este tiempo, para que los estudiantes se den cuenta de sus fortalezas para lograr su aprendizaje, al final del curso se les aplica el postest para corroborar si hay cambios, modificaciones o permanencia en sus estilos de aprendizaje.

\section{RESULTADOS}

Se presenta el análisis de resultados de la encuesta aplicada considerando la clasificación del estilo Honey y Alonso, también se aplicó el test de Sistema de Representación Favorito (de acuerdo al Modelo PNL) a los estudiantes que ingresaron en 2018 a la licenciatura de Químico Farmacéutico Biólogo con la finalidad de obtener información, de la forma en que los estudiantes perciben los conocimientos en el aula. 
Se presentan los siguientes resultados en la tabla 1 y 2 :

Tabla 1 Modelo de Honey - Alonso

\begin{tabular}{|c|c|c|c|c|}
\hline & \multicolumn{2}{|c|}{2018 Pretest } & \multirow[b]{2}{*}{ Curso } & \multirow{2}{*}{$\begin{array}{c}2019 \text { Postest } \\
\text { Estilo de } \\
\text { aprendizaje }\end{array}$} \\
\hline No. & Género & $\begin{array}{l}\text { Estilo de } \\
\text { aprendizaje }\end{array}$ & & \\
\hline 1 & $\mathrm{~F}$ & Reflexivo & 1.- Teoría y ejemplos & Reflexivo \\
\hline 2 & $\mathrm{~F}$ & Reflexivo & 2.- Ejercicios del Modelo Honey -Alonso & Reflexivo \\
\hline 5 & $\mathrm{~F}$ & Reflexivo & $\begin{array}{l}\text { 3.- Durante todo el curso escolar se les } \\
\text { proporciona ejemplos para identificar su canal } \\
\text { preferente }\end{array}$ & Reflexivo - Teórico \\
\hline 8 & $\mathrm{~F}$ & Reflexivo & & Teórico \\
\hline 15 & $\mathrm{~F}$ & Reflexivo & & Reflexivo \\
\hline 16 & $\mathrm{~F}$ & Reflexivo & & Activo-teórico \\
\hline 18 & $\mathrm{~F}$ & Reflexivo & & Teórico \\
\hline 23 & $\mathrm{~F}$ & Reflexivo & & Reflexivo \\
\hline 12 & $M$ & Reflexivo & & Reflexivo \\
\hline 13 & M & Reflexivo & & Reflexivo \\
\hline 14 & $M$ & Reflexivo & & Reflexivo \\
\hline 19 & $M$ & Reflexivo & & Reflexivo \\
\hline 25 & $\mathrm{M}$ & Reflexivo & & Reflexivo - Teórico \\
\hline 27 & M & Reflexivo & & Pragmático \\
\hline 3 & M & Pragmático & & Activo -pragmático \\
\hline 4 & M & Pragmático & & Activo -pragmático \\
\hline 24 & M & Pragmático & & Reflexivo-pragmático \\
\hline 9 & M & Activo & & $\begin{array}{l}\text { Reflexivo-teórico } \\
\text {-pragmático }\end{array}$ \\
\hline 11 & $\mathrm{~F}$ & Teórico & & Reflexivo-teórico \\
\hline 17 & $\mathrm{~F}$ & Teórico & & Reflexivo \\
\hline 10 & M & Teórico & & Reflexivo-teórico \\
\hline 20 & M & Teórico & & $\begin{array}{l}\text { Activo-reflexivo- } \\
\text { pragmático }\end{array}$ \\
\hline 22 & M & Teórico & & Activo-reflexivo \\
\hline 26 & $\mathrm{M}$ & Teórico & & Teórico pragmático \\
\hline 6 & $\mathrm{~F}$ & Reflexivo-teórico & & Reflexivo -pragmático \\
\hline 21 & M & Reflexivo-teórico & & Reflexivo -pragmático \\
\hline 7 & $\mathrm{~F}$ & $\begin{array}{l}\text { Reflexivo- } \\
\text { pragmático }\end{array}$ & & Pragmático \\
\hline
\end{tabular}


La tabla 1 muestra que antes del curso 24 estudiantes aparentemente tuvieron un estilo preferencial específico considerando la clasificación de Honey y Alonso también llamado CHAEA: 8 alumnas reflexivas, 6 alumnos reflexivos, 3 alumnos pragmáticos, 2 alumnas teóricas, 4 alumnos teóricos, 1 alumno activo y sólo dos presentan la combinación; 1 alumno reflexivo -teórico y,1 alumna reflexiva-teórica, 1 alumna la combinación reflexiva-pragmática. Después del curso solo 13 estudiantes se mantuvieron en un solo canal preferencial 5 alumnas reflexivas, 4 alumnos reflexivos, 1 alumna pragmática, 1 alumno pragmático, 2 alumnas teóricas, y los otros 14 reconocieron la utilización de dos canales, 1 alumna activateórica,2 alumnos activos- pragmáticos, 2 alumnas reflexivas-teóricas, 2 alumnos reflexivos - teóricos, 1 alumna reflexiva-pragmática, 2 alumnos reflexivos - pragmáticos , 1 alumno activo- reflexivo, 1 alumno teórico-pragmático, 1 alumno reflexivo-teórico-pragmático y 1 alumno activo- reflexivo-pragmático.
Si bien las preferencias en el estilo de aprendizaje puede mantenerse, también es cierto que el estudiante va adquiriendo nuevas experiencias que le hacen tomar en cuenta habilidades que están ahí en espera de ser utilizadas y solo necesitan la observación dirigida de un docente para que esta emerja, tampoco se puede perder de vista que se trabaja con adolescentes que están experimentando cambios no solo físicos sino también cognitivos, sobre todo porque al estar en un nivel superior de educación, los hace ser más responsables para consigo mismos y dejan de verse como alumnos " $x$ " para comenzar a verse como futuros profesionales de los cuales dependerán otras personas, por ello se observa que el perfil reflexivo se mantiene pero se incrementa el pragmático y el activo así como el teórico, áreas a las que estos estudiante se dirigirán en un futuro. 
Tabla 2 Modelo de PNL (VAK)

\begin{tabular}{|c|c|c|c|c|}
\hline & \multicolumn{2}{|c|}{2018 Pretest } & \multirow[b]{2}{*}{ Curso } & \multirow{2}{*}{\begin{tabular}{|r|}
2019 Postest \\
$\begin{array}{r}\text { Estilo de } \\
\text { aprendizaje }\end{array}$ \\
\end{tabular}} \\
\hline No. & Género & $\begin{array}{c}\text { Estilo de } \\
\text { aprendizaje }\end{array}$ & & \\
\hline 8 & $F$ & Visual & 1.- Teoría y ejemplos & Auditivo \\
\hline 18 & $\mathrm{~F}$ & Visual & 2.- Ejercicios del Modelo VAK & Kinestésico \\
\hline 25 & $\mathrm{M}$ & Visual & $\begin{array}{l}\text { 3.- Durante todo el curso escolar } \\
\text { se les proporciona ejemplos para } \\
\text { identificar su canal preferente }\end{array}$ & $\begin{array}{l}\text { Visual-auditivo- } \\
\text { kinestésico }\end{array}$ \\
\hline 7 & $\mathrm{~F}$ & Auditivo & & Visual \\
\hline 17 & $\mathrm{~F}$ & Auditivo & & Auditivo \\
\hline 10 & $M$ & Auditivo & & Kinestésico \\
\hline 12 & $M$ & Auditivo & & Auditivo \\
\hline 20 & $M$ & Auditivo & & Auditivo \\
\hline 22 & $\mathrm{M}$ & Auditivo & & Auditivo - kinestésico \\
\hline 27 & $\mathrm{M}$ & Auditivo & & Visual- auditivo \\
\hline 5 & $\mathrm{~F}$ & Kinestésico & & Auditivo \\
\hline 11 & $\mathrm{~F}$ & Kinestésico & & Auditivo \\
\hline 3 & $\mathrm{M}$ & Kinestésico & & Auditivo - kinestésico \\
\hline 4 & $M$ & Kinestésico & & Kinestésico \\
\hline 9 & $M$ & Kinestésico & & Auditivo - kinestésico \\
\hline 14 & $M$ & Kinestésico & & Visual \\
\hline 1 & $\mathrm{~F}$ & Visual- auditivo & & Visual \\
\hline 2 & $F$ & Visual- auditivo & & Auditivo \\
\hline 13 & $F$ & Visual- auditivo & & Visual- auditivo \\
\hline 15 & $F$ & Visual- auditivo & & Auditivo - kinestésico \\
\hline 19 & $M$ & Visual- auditivo & & Auditivo \\
\hline 6 & $F$ & $\begin{array}{llllll} & \text { i } & \text { s } & \text { u } & \text { a } & \text { I } \\
\text {-Kinestésico }\end{array}$ & & Kinestésico \\
\hline 21 & $\mathrm{M}$ & $\begin{array}{l}\mathrm{V} \text { i s u } \quad \text { a } \\
\text {-Kinestésico }\end{array}$ & & Visual- auditivo \\
\hline 24 & $\mathrm{M}$ & $\begin{array}{llllll}V & \text { i } & \text { } & \text { u } & \text { a } & \text { I } \\
\text {-Kinestésico } & \end{array}$ & & Auditivo \\
\hline 16 & $F$ & $\begin{array}{l}\text { A u d i t i v o- } \\
\text { Kinestésico }\end{array}$ & & Auditivo \\
\hline 23 & $F$ & $\begin{array}{l}\text { A u d i t i v o- } \\
\text { Kinestésico }\end{array}$ & & Visual - kinestésico \\
\hline 26 & $M$ & $\begin{array}{l}\text { Visual-auditivo - } \\
\text { Kinestésico }\end{array}$ & & Auditivo \\
\hline
\end{tabular}


La tabla 2 muestra que antes del curso 16 estudiantes aparentemente tuvieron un estilo preferencial específico considerando la clasificación Respecto al canal Visual, Auditivo, Kinestésico, también llamado VAK 2 alumnas visuales , 1 alumno visual, 2 alumnas auditivas, 5 alumnos auditivos ,2 alumnas kinestésico ,4 alumnos kinestésicos, solo 11 presentaron la combinación 2 alumnas auditivo - kinestésico, 3 alumnas visual - auditivo ,2 alumnos visual -auditivo, 1 alumna visual - kinestésico, 2 alumnos visual -kinestésico ,1 alumno auditivo - visual - kinestésico, después del curso 18 estudiantes tuvieron un canal preferencial: 2 alumnas visuales ,1 alumno visual, 6 alumnas auditivas, 5 alumnos auditivos, 2 alumnas kinestésicas ,2 alumnos kinestésicos, solo 9 presentaron la combinación 1 alumna auditiva - kinestésica, 3 alumnos auditivos -kinestésicos, 3 alumnos visuales - auditivos, 1 alumna visual - kinestésica, 1 alumno visual - auditivo kinestésico.

Al igual que el instrumento anterior se observa la modificación o mantenimiento del canal preferencial del estudiante, algunos se vuelven más activos, o kinestésicos, y otros descubren que usan la audición más de lo que ellos creían, y aparentemente el canal visual disminuye, no se puede dejar de lado que estamos tan inmerso en las TIC'S, que la vista es un sentido que ya no se considera como algo preferencial por estar tal vez saturados de tanta información visual.

\section{DISCUSIÓN}

Se observan cambios, aunque el canal preferencial en algunos estudiantes no cambia, permanece, en otros cambia, ya que utiliza otros canales fortaleciendo su aprendizaje. Esto no se debe a que los estudiantes hayan decidido cambiar, sino que ahora reconocen que hacen uso de diferentes canales, herramientas y formas de aprender, ya que la forma de aprender cambio en los estudiantes con el uso de la tecnología que prácticamente ha obligado a estos jóvenes, este es un nuevo reto para los docentes acostumbrados a trabajar con un solo estilo de enseñanza basado por lo general en su propio estilo de aprendizaje. Se observo durante el curso la conducta de los visuales como organizados, ordenados, observadores y tranquilos. de los auditivos fue que se distraen fácilmente, mueve los labios al leer, facilidad de palabra, no le preocupa especialmente su aspecto, y de los kinestésicos se observó

que responden a las muestras físicas de cariño, les gusta tocarlo todo, se mueven y gesticulan mucho expresando sus emociones con movimientos.

Por lo que el facilitador actual, debe de considerar estos estilos para diseñar estrategias eficaces para que los estudiantes, analicen su propio proceso de aprendizaje, fortaleciendo su proceso de "aprender a aprender".

\section{CONCLUSIÓN}

Se requiere que los estudiantes reconozcan sus diferentes estilos de aprendizaje y sus canales de percepción que tienen, así como utilizarlos, con el acompañamiento del facilitador para fortalecer su aprendizaje, al utilizar actualmente estrategias novedosas que favorecen el uso de las tecnologías para este proceso, fortalece su aprendizaje durante su trayectoria académica.

En este estudio se demuestra que es importante continuar trabajando con los estudiantes de nuevo ingreso ya que al reconocer sus estilos de aprendizaje les ayudará a su proceso de aprendizaje en la trayectoria académica en el nivel superior.

También les permite a los estudiantes, darse cuenta de las diversas habilidades que pueden mejorar si utilizan su canal preferencial y su estilo de pensamiento, así como establecer un mejor trabajo colaborativo con sus compañeros de clase que comparten sus preferencias de aprendizaje y por supuesto el docente, mejora 
su papel de facilitador cuando hace uso de la detección oportuna de los estilos y preferencias de sus estudiantes, lo que redunda en un mejor aprovechamiento escolar.

\section{BIBLIOGRAFÍA}

Alonso, C., Gallego, D., Honey P. (1994). Los estilos de aprendizaje. Procedimientos de diagnóstico y Mejora. Ed. Mensajero. Bilbao, España.

ANUIES. (2006). Antología del curso estilos de aprendizaje y estrategias de enseñanza. Universidad Autónoma de Campeche. México.

Castillo Díaz Marcio Alexander; Mendoza Aly, Jorge Luis. (2015). Estilos de aprendizaje en estudiantes universitarios: recursos informáticos como estrategia para su evaluación. UNAH INNOV@ No 42015 pág. 33-39 digital.

Domínguez Rodríguez, Heriberto de Jesús ; Gutiérrez Limón, Jorge Alberto; Llontop Pisfil , Manuel; Villalobos Torres, David; Delva Exume, Jean Claude.( 2015). Estilos de aprendizaje: un estudio diagnóstico en el centro universitario de ciencias económico-administrativas de la U de G. Revista de la Educación Superior. ANUIES. Volumen 44. Pp 121140.

Escanero-Marcén, Jesús F., Soria, M. Soledad, Guerra-Sánchez, Manuel, \& Silva, Jackson. (2016). Comparación de los estilos de aprendizaje de los alumnos de medicina obtenidos con un nuevo cuestionario con los proporcionados por el cuestionario Honey-Alonso (CHAEA). FEM: Revista de la Fundación Educación Médica, 19(1), 19-26. Recuperado en 14 de octubre de 2019, de http://scielo.isciii.es/scielo. php?script=sci_arttext\&pid=S2014$98322016000100006 \&$ Ing=es\&tlng=es.

Gutiérrez Tapias, Mariano, (2018). Estilos de aprendizaje, estrategias para enseñar. Su relación con el desarrollo emocional y "aprender a aprender". TENDENCIAS PEDAGÓGICAS N³1 2018. España. P .94 .

González Garza, Beatriz, Hernández Castañón, Ma. Alejandra, \& Castrejón Reyes, Victorina. (2018). Estilos de aprendizaje para el desarrollo de competencias en estudiantes de la Licenciatura en Enfermería. RIDE. Revista Iberoamericana para la Investigación y el Desarrollo Educativo, 8(16), 351369. https://dx.doi.org/10.23913/ride. v8i16.345.

Neira Silva J. (2015). Visual, Auditivo o kinestésico los alumnos. Orientación Andújar. Perú.

Reyes, L., Céspedes, G., Molina, J. (2017). Tipos de aprendizaje y tendencia según modelo VAK. TIA, 5(2), Bogotá -Colombia. pp. 237-242.

Robles, Ana. (2000). Estilos de aprendizaje: como seleccionamos y representamos la información. Disponible en http:// www.galeon.com/aprenderaaprender/ general/indice.html.(consultado en 2012). México. 